\title{
$1 / f$ neural noise is a better predictor of schizophrenia than neural oscillations
}

Abv. title: Neural noise predicts schizophrenia

Erik J. Peterson ${ }^{\star c}$, Burke Q. Rosen ${ }^{a}$, Alana M. Campbell ${ }^{d}$, Aysenil Belger $^{d}$, Bradley Voytek ${ }^{a, b, c}$

${ }^{a}$ Neurosciences Graduate Program, Institute for Neural Computation, 'Department of Cognitive Science, University of California, San Diego; 9500 Gilman Drive, La Jolla, 6 CA 92093, United States. ${ }^{d}$ Departments of Psychiatry and Psychology, University of North Carolina at Chapel Hill, Chapel Hill, NC 27599, United States.

$E P$ and $B V$ designed the research. $E P, B R$, and $A C$ analyzed the data. $E P, B V, A C$ and $A B$ wrote the paper.

Correspondence should be addressed to Erik Peterson. Email: e7peterson@ucsd.edu

Number of Figures: 3

Number of Tables: 1

Number of Multimedia: 0

Number of words for Abstract: 57

Number of words for Sig. Statement: 108

Number of words for Introduction: 566

Number of words for Discussion: 1296

Acknowledgements: None

The authors declare no competing financial interests. 


\title{
$1 / f$ neural noise is a better predictor of schizophrenia than neural oscillations
}

\begin{abstract}
Schizophrenia has been associated with separate irregularities in several neural oscillatory frequency bands, including theta, alpha, and gamma. Our multivariate classification of human EEG suggests that instead of irregularities in many frequency bands, schizophrenia-related electrophysiological differences may better be explained by an overall shift in neural noise, reflected by a change in the $1 /$ f slope of the power spectrum.
\end{abstract}

\section{Significance statement}

Understanding the neurobiological origins of schizophrenia, and identifying reliable biomarkers, are both of critical importance in improving treatment of that disease. While we lack predictive biomarkers, numerous studies have observed disruptions to neural oscillations in schizophrenia patients. This literature has, in part, lead to schizophrenia being characterized as disease of disrupted neural coordination. We report however that changes to background noise (i.e., 1/f noise) are a substantially better predictor of schizophrenia than both oscillatory power and participants own behavioral performance. The observed alterations in neural noise are consistent with inhibitory neuron dysfunctions associated with schizophrenia, allowing for a direct link between noninvasive EEG and neurobiological deficits. 


\section{Introduction}

Schizophrenia is characterized by disruptions to multiple cognitive and behavioral domains, including significant working and long-term memory disruptions, impaired attention, and disorganized patterns of thought and language. These cognitive deficits are in addition to the well-established delusions, hallucinations, and affective blunting (Uhlhaas et al, 2010; Crespo-Facorro et al., 2001). Abnormalities in individual brain regions, or cognitive systems, cannot explain the complex and varied schizophrenic symptomatology. Instead, widespread physiological abnormalities leading to reduced and disorganized neural communications are thought to be a better predictor of schizophrenic pathophysiology (Phillips \& Silverstein, 2003; Uhlhaas et al, 2010, 2012).

Two specific deficits-increases in neural response variability (i.e., neural noise), and disruptions to oscillatory coupling-are thought to be especially critical for the onset and severity of deficits in schizophrenia (Uhlhaas et al, 2010, 2012). There is ample evidence that disruptions to multiple oscillatory bands are associated with schizophrenia. Disruptions in neural oscillatory activity in schizophrenia may explain deficits in both perceptual processing and disorganized thought patterns (Uhlhaas et al, 2010). Electrophysiological investigations of schizophrenia typically report that power in the low frequency delta $(1-3 \mathrm{~Hz})$ and theta $(4-8 \mathrm{~Hz})$ bands increases, power in the mid-frequency bands of alpha $(8-12 \mathrm{~Hz})$ and beta $(12-30 \mathrm{~Hz})$ is unchanged, while power in the gamma range $(>30 \mathrm{~Hz})$ is decreased (Uhlhaas et al, 2010, Sun et al, 2011). This pattern of activity is typically explained as a series of band specific changes, e.g. theta increases independently of the decrease in gamma. 
While these oscillatory results have proved to be robust, there is scarce direct evidence regarding neural noise changes in schizophrenia, as measuring neural noise has proved to be more difficult. Recently, however, it has been proposed that neural noise can be estimated from the EEG power spectrum (Voytek et al 2015). Specifically, noise in the spectral domain can be clearly identified by its characteristic $1 / f^{\chi}$ (power-law) shape. That is, power in a spectrogram decreases exponentially as a function of frequency, where the exponent $\chi$ determines the 'slope' of the decline. Of particular interest is that fact that, traditionally, oscillations are measured assuming that this $1 / \mathrm{f}$ noise is fixed. Emerging evidence however suggests slope can vary in meaningful ways (El Boustani et al., 2009, Freeman \& Zhai, 2009, Podvalny et al., 2015, Voytek \& Knight, Voytek, B. 2015). For example, healthy aging is associated with an increase in neural noise (Salthouse \& Lichty, 1985) and with changes in the "slope" of the 1/F power spectrum (Voytek, et al, 2015, Wöstmann et al, 2017). This noise perspective is particularly intriguing from an information theoretic perspective, where information theory suggests that increasing noise fundamentally decreases the efficiency of communications (Shannon, 1948). Consistent with this, schizophrenia has been previously linked to decreases in neural signal-to-noise (Winterer \& Weinberger, 2003).

Modeling studies suggest neural noise and oscillatory coupling are interdependent. Increasing noise can destabilize neural oscillations (Wang, X.-J., 2010). Likewise, variations in oscillatory power and phase can modulate background excitability (Womelsdorf et al., 2014) and therefore alter noise. Thus, based on prior reports linking schizophrenia symptomatology to abnormal neural noise, we hypothesized that schizophrenic patients should exhibit abnormal power spectrum slopes compared to healthy controls. We further hypothesized that measurements of band-limited oscillatory power may be confounded by changes in background 
electrophysiological noise. To begin to separate these factors we undertook multivariate analysis of EEG data to identify whether neural noise or oscillatory power was the most reliable predictor of schizophrenic clinical status.

\section{Methods}

\section{Participants}

EEG and behavioral data were acquired for 65 (1 left-handed) participants recruited from two groups: schizophrenia patients ( $n=31,7$ female, age range $19-38$ ) or matched control participants $(n=35,16$ female, age range: 19-41). Schizophrenia patients were referred by their treating clinician or were recruited from the community alongside the control participants. Patients met the DSM-IV criteria for schizophrenia-spectrum illness using the SCID interview (First et al, 2002). To reduce long term effects of medication, only recent-onset patients were studied, with onset occurring within the last five years. Schizophrenia patients were stable and continued treatment plans throughout the course of the study. Study procedures were approved by the Institutional Review Board of [Author University]. Participants were compensated for their participation.

\section{Experimental protocol}

Data were recorded from a standard 10-20 electrode montage 32-channel EEG cap (Electo-Cap International) using NeuroScan 4.3.1 software and a SynAmps amplifier. Continuous EEG data were sampled at $500 \mathrm{~Hz}$ with a $0.15-70 \mathrm{~Hz}$ band-pass filter and $60 \mathrm{~Hz}$ notch filter. Electrode AFz served as ground and the right mastoid as reference. Vertical and 
horizontal bipolar electroocculogram (EOG) electrodes were placed at the outer canthi of each eye and above and below the right eye. Electrode sites were exfoliated and contacts were affixed with conductive gel. Electrode impedance was kept below $5 \mathrm{k} \Omega$. Recordings were acquired in sound-dampened chamber with participants seated $80 \mathrm{~cm}$ from the stimulus presentation monitor.

\section{Task}

EEG was recorded while participants completed a selective attention task (see Figure 1). Stimuli consisted of a letter $(\mathrm{E}, \mathrm{H}$, L, or $\mathrm{P})$ or number $(3,7,8$, or 9$)$ presented in either red or green typeface on a black background. Each participant was instructed to identify a target stimulus of the correct alphanumeric class and presented in the correct color. Letters were targets (numbers, non-targets) and only one indicated color required attention. Thus, stimuli were binned into four trial conditions: attended targets (letter stimuli of the indicated color), attended non-targets (numeric stimuli of the indicated color), non-attended targets (letter stimuli of the non-indicated color), and non-attended non-targets (numeric stimuli of the non-indicated color). All bins occurred with equal frequency (25\%). Stimuli were presented for $200 \mathrm{~ms}$ with a jittered inter-stimulus interval of $1500 \pm 200 \mathrm{~ms}$, during which a white fixation cross was presented. Participants performed 5 blocks of 100 stimuli each for a total of 500 trials. Overall, 125 trials of each condition were presented in a pseudorandom order. Participants were instructed to respond to attended target stimuli with the left mouse button (index finger) and to all other stimuli with the right mouse button (middle finger). Early analyses showed that the attended non-target, non-attended target, and non-attended non-target conditions exhibited similar behavioral and physiological responses. Therefore, data from these conditions was 
combined by drawing equal numbers of trials at random from each of the three conditions such that the total count matched the number of attended trials for each participant. In subsequent analyses, the combined non-attended target condition trials were compared to attended trials.

\section{Data analysis}

Continuous EEG data were segmented into $500 \mathrm{~ms}$ epochs following initial stimulus presentation. Epochs containing artifacts (as determined by a $\pm 100 \mu \mathrm{V}$ threshold or manual inspection) were removed and eye-blink components identified via independent component analysis (ICA) (Makeig et al. 1996; Jung et al. 2000) were removed from further analyses. Artifactual components were selected manually and confirmed by monitoring for reduced correlations between post-ICA EEG and EOG waveforms. All subsequent analyses were performed independently for each EEG channel. Baseline activity was removed by subtracting mean pre-stimulus voltage from each epoch. Participant performance was quantified by computing accuracy and response times (RTs).

Frequency domain analyses were performed by first computing the power spectral density (PSD) of each artifact-free epoch using Welch's method (Welch 1967). For each participant and channel, the PSDs of all epochs of each condition were averaged together. Traditional PSD analysis was performed by dividing the spectra into the following canonical bands: theta $(4-8 \mathrm{~Hz})$, alpha $(8-12 \mathrm{~Hz})$, beta $(12-30 \mathrm{~Hz})$, and gamma (30-50 Hz). In addition, the slope, or exponential decay parameter $\chi$ of each average spectrum was estimated using robust linear regression algorithm (i.e. RANSAC; Fischler \& Bolles, 1981) across the 4-50 Hz range. In this method, the underlying $1 / f$ framework of the spectrum is treated as linear in log-log space 
(see eq. 1). In this method oscillatory peaks in the spectrum are treated as outlier deviants from this pattern and thus removed using a robust linear model (Fischler and Bolles 1981). The spectral power law is modeled by the log-linear decay coefficient $\chi$, where $f$ is frequency and $S(f)$ is power (Eq. 1).

$$
\log (S(f))=\chi * \log (f)
$$

Statistical analysis

Analysis of the distribution of both behavioral and electrophysiological data revealed the majority of the data was significantly skewed (i.e., behavioral accuracy, as well as all power measurements). We therefore adapted our summary statistics and our statistical methodology to use robust measures, those less susceptible to bias by extreme values. Central tendency is reported using the median, not the customary mean. Variance was likewise measured using the median absolute deviation (MAD; MAD $=\operatorname{median}\left(\left|x_{i}-\operatorname{median}(X)\right|\right)$, where $X$ is represents the complete sample and $x_{i}$ represents individual samples). Pairwise null hypothesis testing was conducted using Wilcoxon rank sum test. All statistical analysis was done using the $\mathrm{R}$ programming language ( $\mathrm{R}$ Core Team, 2016. R.). Analysis code, and raw data is available at [available post-publication].

\section{Multivariate classification}

We used multivariate classification to predict patient status (schizophrenia or control) in a selective attention dataset. We compared three different feature sets (behaviour, oscillatory band power, slope) and a range of classifiers (discussed below). To ensure a rigorous test of 
general model performance, prior to undertaking any multivariate analysis $30 \%$ of both neural and behavioral data was randomly selected as a "holdout", or final validation, set. Modeling of this set occurred only after model selection (described below) was complete. Additionally, all normalization and rescaling of features used parameters derived separately for holdout or training sets, ensuring independence. That is, the holdout datasets were tested only on a single model per condition. We report only holdout performance here.

An estimate of the precision of classifier accuracy and an estimate of null performance (i.e., shuffled label performance) are useful in assessing overall classifier performance. To estimate both, we conducted two bootstrap procedures. To estimate chance performance we randomized and resampled (with replacement) the group labels (CO or SZ), generating a null distribution (see grey bars in Figure 3). Our second bootstrap procedure left the labels intact but randomly sub-sampled the electrode averaged data (with replacement). This sampling procedure allowed us to better estimate the accuracy variance inherent to the holdout data set. Of course, the single random selection event inherent in creating a holdout set may have been accidentally biased, compared to some true, but unknowable, value. The resampling procedures used here do not remove this potential (and uncontrollable) source of bias. However given that we report the median values of these resampling procedures, the effect of extreme bias events should be minimized. Bootstrapping distributions all consisted of 500 independent samples.

Prior to running the final holdout analysis, we undertook an extensive model and feature selection procedure. Model selection, using $70 \%$ of the data, relied on 10 -fold cross validation (CV), as well as bootstrapping analysis similar to that outline above. We considered a range of modeling approaches ranging from OLS-based logistic regression to nonlinear models taking 
advantage of both concurrent strong regularization, and/or boosting (e.g., Bagged AdaBoost, Boosted Logistic Regression, SVM w/ either radial or polynomial kernels, and Ridge Regression). In the end, we selected a linear support vector machine (linear SVM) for behavioral classification, and nonlinear 'Random Forests' for classifying both types of neural data - spectral power and slope. Applying linear models to the neural data tended to reduced performance in the training set by $5-10 \%$ (not shown). Likewise, applying a range of nonlinear models (see above) to the behavioral data strongly negatively impacted performance (>10\%). We reasoned then that it was better to maximize classification performance on these different data types rather than enforce a consistent (inappropriate) model across both data types. Hyper-parameter optimization for both models was estimated using only training data, see Table 1. 


\begin{tabular}{|l|l|l|}
\hline Features & Model & $\begin{array}{l}\text { Hyper- } \\
\text { parameters }\end{array}$ \\
\hline Behavior (accuracy, reaction time) & $\begin{array}{l}\text { Linear } \\
\text { SVM }\end{array}$ & $\mathrm{C}=0.25$ \\
\hline $1 /$ f spectral slope & $\begin{array}{l}\text { Random } \\
\text { Forest }\end{array}$ & $\mathrm{mtry}=9$ \\
\hline Oscillatory power (theta, alpha, beta, gamma) & $\begin{array}{l}\text { Random } \\
\text { Forest }\end{array}$ & $\mathrm{mtry}=2$ \\
\hline Oscillatory power (gamma only) & $\begin{array}{l}\text { Random } \\
\text { Forest }\end{array}$ & $\mathrm{mtry}=6$ \\
\hline
\end{tabular}

Table 1. Classifiers, features design, and cross-validation optimized hyperparameters. All spectral features were taken from 10 central, posterior, and midline EEG electrodes (i.e., C3, C4, Cz, FCZ, Fz, O1, O2, P3, P4, Pz).

\section{Results}

\section{Task accuracy differs in schizophrenia patients}

Schizophrenia patients had significantly lower accuracy $(0.91+/-0.11$ MAD) compared to the control group (0.95 +/- 0.05) (Wilcoxon rank sum test, $\mathrm{W}=2896, p=2.3 e-5)$ (Figure 1b). Both groups had worse performance for attention compared to no-attention trials (control W $=1090.5$, $p=2.24 e-10$, Schizophrenia: $W=859.5, p=9.57 e-08)$. Reaction time was not a significant 
predictor of group membership $(\mathrm{W}=1954, \mathrm{p}=0.4743 ; 525.51+/-95.54$ compared to $528.06+/-$ 100.68). Within groups, reliable reaction time effects of condition were observed such that, in control subjects, the attention condition had significantly faster reaction time $(489.86+/-82.97$ $\mathrm{ms})$ compared to the no attention condition $(578.62+/-88.26 \mathrm{~ms})(\mathrm{W}=278, \mathrm{p}=0.000163)$. Similarly, schizophrenia patients were significantly faster for attention $(467.55+\mid-83.36)$ compared to no attention trials $(562.5+/-77.6 \mathrm{~ms})(\mathrm{W}=246, \mathrm{p}=0.0007666)$ (Figure 1c). 
a

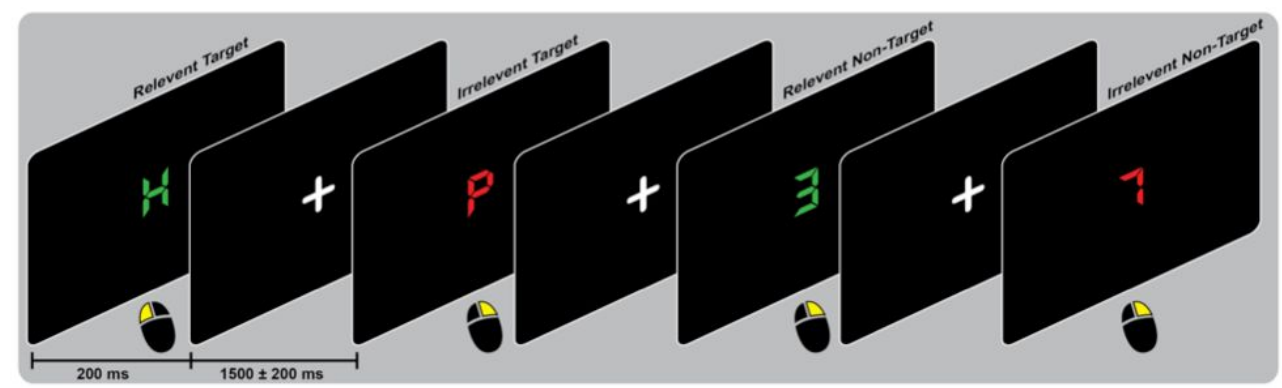

b

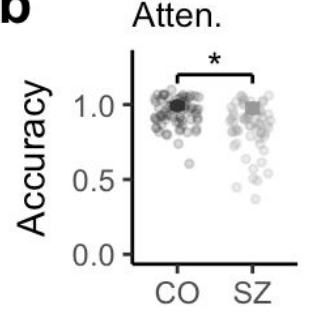

No atten.

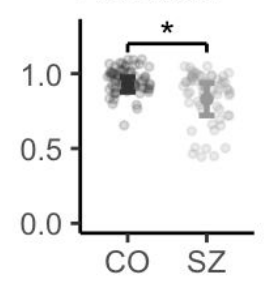

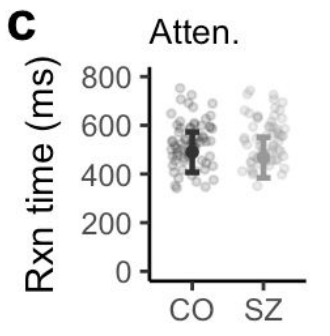

No atten.

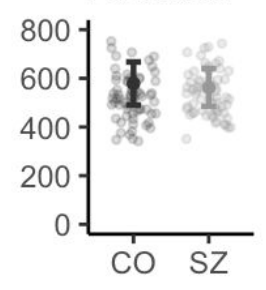

Figure 1. Task and behavior results. a) Task diagram. Comparison of median (b) accuracy and c) reaction time (+/- MAD), between the two groups (control [CO] and schizophrenia [SZ]) and task conditions ([atten]tion and [no atten]tion).

\section{Band-specific increases and decreases in oscillatory power}

In the theta band there were no significant there power differences between control and schizophrenia (Wilcoxon rank sum test, $W=17268, p$-value $=0.1255$ ), consistent with the small increase in median power difference in $(3.49+/-2.13)$ compared to controls $(3.19+/-1.98)$ (Figure 2b). In the alpha range, schizophrenia patients showed significantly elevated power $(2.39+/-1.64)$ compared to control $(1.96+/-1.38)(\mathrm{W}=16068, \mathrm{p}$-value $=0.0090)($ Figure $2 \mathrm{c})$ while controls had significantly less median power $(0.38+/-0.18)$ than patients in the beta range 
$(0.47+/-0.26)(\mathrm{W}=15250, \mathrm{p}$-value $=0.00082)($ Figure $2 \mathrm{~d})$. In the gamma band, however, the

trend reversed. Power was significantly reduced in patients $(0.11+/-0.07)$ versus controls $(0.12$

+/- 0.05) $(\mathrm{W}=21492, \mathrm{p}$-value $=0.02346)($ Figure 2e $)$.

1/f spectral slope decreases in schizophrenia and best predicts schizophrenia status

The slope of the power spectrum in schizophrenia patients was significantly steeper $(1.94+/-$

$0.48 \mathrm{MAD})$ compared to controls $(1.68+/-0.55)$ (Wilcoxon rank sum test, $\mathrm{W}=14104, \mathrm{p}=$ 1.2e-05) (Figure 2f-h). 

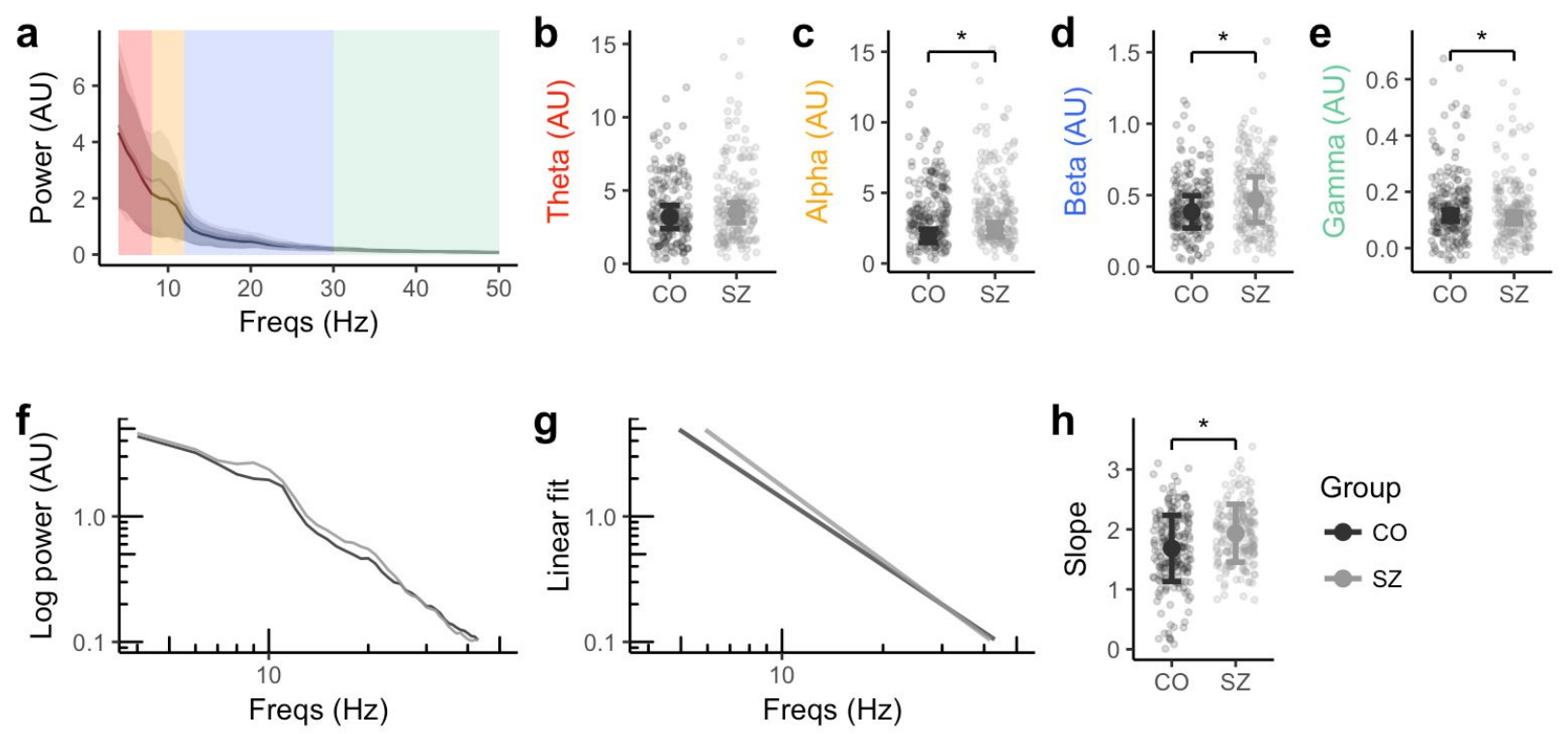

Figure 2. Oscillatory power and spectral slope in Schizophrenia patients (grey) and controls (black). a) Median spectral power spectrum (MAD error in represented by shaded areas). Color coded regions represent oscillatory bands - theta (red, 4-8 Hz), alpha (orange, 8-12 Hz), beta (blue, 12-30 Hz), gamma (green, 30-50 Hz). b-e) Median power for each band (+/-MAD). f) Log transformed spectral power. g) Example robust linear fitting of the slope of (e). h) Median slope differences between groups (+/- MAD).

Spectral power varies between cortical regions and can be used to identify individual subjects, and tasks (Cox, R. et al., 2016). We therefore employed a spatial multivariate classification approach to predict clinical group (i.e., control or schizophrenia) from either oscillatory band power, spectral slope, or both (see Methods). We contrasted these outcomes with predictions made using behavioral data. 
The classification model based on spectral slope (Figure 3b) achieved the highest median classification accuracy $(0.71+/-0.11 \mathrm{MAD})$ compared to a model using all oscillatory band data $(0.57+/-0.11)$ (Figure $3 c)$. Slope also outperformed gamma power alone $(0.57+/-$ 0.11) (Figure 3d). Further, a joint model relying on slope and all oscillatory bands showed reduced performance $(0.64+/-0.11)$ compared to slope alone (median difference of 0.07 ; Figure 3e). Such a reduction is expected if slope and band power are collinear, or otherwise highly covariate. The model based on behavioral response (i.e., reaction time and response accuracy) performed better than either of the oscillatory models $(0.67+/-0.12$; compare Figure 3a to c-e). Behavioral predictive performance was, however, inferior to spectral slope (0.67 compared to 0.71; Figure 3a to $\mathbf{b})$. 

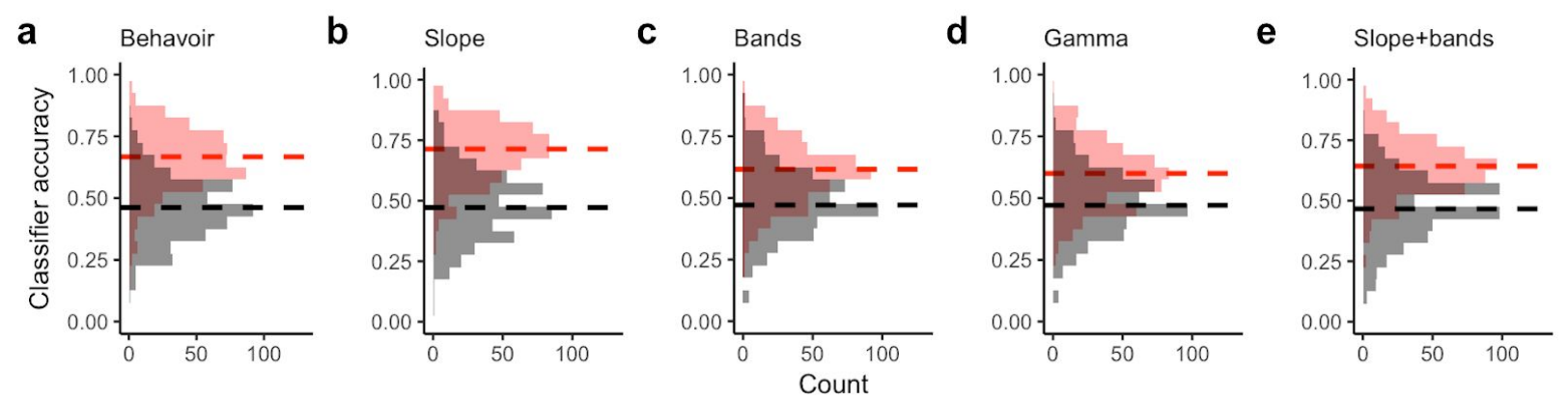

Figure 3. Classifier performance. Classification accuracy was assessed on a hold out data set, comprising $30 \%$ of each data set. The effect size, i.e. performance above chance, was estimated by comparing hold out performance to a label-shuffled null distribution (grey). Model precision was estimated by subjecting the holdout set to a bootstrapping procedure (red). Median performance indicated by dashed lines. a) Classification accuracy using only behavioral data b) Performance using spectral slope c) Accuracy for all four canonical oscillatory bands (theta, alpha, beta, gamma, see Figure 2a). d) Accuracy using only gamma band power e) A joint model, including both spectral slope and all oscillatory bands.

\section{Discussion}

Our findings reveal that spectral slope, a measure of the neural background noise, is a better predictor of schizophrenia than power measurements derived from specific oscillatory bands or from behavioral responses collected during a selective attention task. The latter is an especially surprising result considering that behavioral measurements remain the gold standard in classifying clinical outcomes in schizophrenia. Given the increasing demand for more 
objective biomarkers to identify and classify clinical pathology, we suggest that spectral slope may serve as an effective biomarker for schizophrenia.

\section{Oscillatory power differences in context}

The direction and significance in band-specific differences observed in the current report are broadly consistent with prior work (see Figure $2 \mathbf{a}$ ). The only deviating result is the lack of a group difference in theta power. However all other bands displayed a significant separation based on clinical status.

There is ample evidence that gamma activity is disrupted in schizophrenia. Interpreting these disruptions, however, is difficult. Current results suggest gamma oscillations are highly stimulus, task, and context dependent (Merker, B., 2013; Ray et al 2010; Hermes et al 2014), though it is important to note that power in the gamma band may not always reflect the presence of a true oscillation in that band. However even when present, the power of gamma oscillations fluctuates strongly in time, and is suppressed by task-irrelevant factors (Merker, B., 2013, Ray et al 2010). We interpret the fact that gamma-alone was a poorer predictor of disease status than slope to be due in part to this variability. We additionally note that there was a strong alpha peak present in the power spectra in our data (Figure 2f). Like gamma, this difference was significant, however the strength alpha's predictive capacity using spatial variations in power (a classification accuracy of $0.56+/-0.12)$ was still well below that of spectral slope $(0.71+/-0.11)$ and also well below behavior-based predictions $(0.65+/-0.12)$. 


\section{Slope and oscillatory power may be complementary}

In this selective attention dataset we observed that spectral slope, a measure of neural background noise, better predicts schizophrenia compared to band-specific power changes. Combined with previous results in cognitive aging (Voytek et al, 2015), we argue that neural noise, as indexed via spectral slope, can serve as a useful biomarker in clinical populations. The ample literature reporting band specific anomalies in schizophrenia patients complement the range of studies that suggest oscillatory coupling is relevant for healthy cognitive function. However, if our results are representative, future work should consider isolating changes in band-specific power from changes in background noise.

Based on the strength of our classification results, we expect slope to prove a reliable predictor of schizophrenia status outside this single experiment. While we do not expect slope will explain the wealth of band-specific variations across the literature, we believe that measurements of band-limited power and slope may prove to be complementary pieces of information that will improve the utility of electrophysiology as a clinical biomarker. Indeed, both oscillations and noise may be needed to understand, and computationally model, the complex biological interactions that are not doubt involved in generating schizophrenia pathophysiology. For example, an increase in gamma power that occurs inline with a chronic increase in inhibitory conductance (see below) may have an overall suppressive effect on neural excitability (Ray, S et al 2013) whereas in a more homeostatic condition, a gamma can instead act to increase neural gain (Womelsdorf, T. et al., 2014). That is, the functional role of gamma oscillations may change as inhibitory conductance increases. Oscillations with an abnormally strong inhibitory 
may act to gate or suppress information transmission (Ray \& Maunsell, 2013, Börgers \& Kopell, 2008), whereas gamma oscillations operating in a more balanced regime may instead amplify information flow (Womelsdorf, T. et al., 2014).

\section{Steeper slope may reflect increased inhibitory conductance}

The slope of the power spectrum is described by a 1/F power law distribution. We focus here on the range from $\sim 4-50 \mathrm{~Hz}$. In this range, slope $(\chi)$ values vary from to 1-4 (Freeman, W.J. \& Zhai, J., 2009, Podvalny, E. et al., 2015). The larger the $\chi$ the steeper the slope of the power spectrum.

Power laws such as the $1 / f^{\chi}$ electrophysiological power spectrum arise from a large number of physical sources (Kendal, W.S., 2013). In neural electrophysiology, aspects of the power spectral slope have been attributed to shot and brownian noise (Mandelbrot, B.B. \& Van Ness, J.W., 1968, Milstein, J. et al., 2009), and to network connectivity (Freeman, W.J. \& Zhai, J., 2009, El Boustani, S. et al., 2009). Others use the $1 / f^{\chi}$ pattern to suggest the brain is operating like a dynamical system tuned to a self-organized "critical point" (Bak, P., Tang, C. \& Wiensenfeld, K., 1988,Bédard, C., Kröger, H. \& Destexhe,Chaudhuri, R., He, B.J. \& Wang, X., 2016). Most obviously relevant to Schizophrenia however are recent modeling results, which link changes in power spectral properties to changes in synaptic activity (Miller, K.J., 2010) and excitatory and inhibitory currents (Destexhe, A. \& Rudolph, M., 2004). These efforts, which relate synaptic fluctuations in the membrane voltage to local field potentials (LFP), are supported by recent combined recordings of LFP and single units in human subjects showing that slower inhibitory time constants dominate the LFP (Dehghani, N. et al., 2016).. 
In response to these results, we have recently developed a computational model to infer changes in excitatory-inhibitory balance directly from spectral slope in LFP recordings (Gao, et al, 2016). In this model, increasing the inhibitory conductance, or the time constant of inhibitory kinetics, increases slope. Conversely, decreasing the proportion of inhibitory activity, or increasing excitatory conductance, decreases slope. That is, our model suggests the increase in slope we report here may be a direct consequence of increased inhibitory conductances. This increase is presumably a homeostatic response to the decreased number of inhibitory neurons associated with schizophrenia. Within a limit, increasing the strength of individual synapses can compensate for the overall reduction in inhibitory interneurons, however this may reach a pathological tipping point over time.

If the observed increase in spectral slope is caused by a loss of inhibition and the corresponding homeostatic adjustment, this may explain why slope is the superior predictor of disease status. Loss of inhibition appears as a widespread and chronic phenomenon in schizophrenia. That is while excitatory-inhibitory imbalance ultimately affects oscillatory coupling, oscillatory changes depend on a number of external factors (e.g., attention, stimulus contrast, size, task context, goals). These external factors may act as dynamic "confounds" that are shared between control and schizophrenia subjects undertaking the same cognitive task. Meanwhile slope may reflect a more fundamental physiological index - inhibitory conductance allowing for the observed improvement in classification performance. 


\section{Drug effects, and other important caveats.}

We selected schizophrenia patients diagnosed within the last five years in an effort to minimize drug effects contaminating the electrophysiological results. However both the slope and band specific changes we report here may nevertheless be due, perhaps in part, to pharmacological confounds. Controlling for this possibility in a chronic condition like schizophrenia is difficult. Ultimately we will need to confirm these results in drug free individuals and in animal models. However more elaborate experiments require an initial robust result, which is what we report here.

We have reported results for a single selective attention task. Despite our relatively large patient pool $(n=31)$, the results require confirmation in other tasks and, ideally, in other laboratories. Concurrent with the line of research outlined here, we are also investigating $1 /$ f noise changes with healthy cognitive aging. The slope increase we previously reported with age in ECoG data (Voytek et al 2015) have already been replicated by independent researchers (Waschke et al, 2017). We are therefore optimistic that the schizophrenia related $1 / \mathrm{f}$ changes reported here will prove similarly robust. 


\section{References}

Bak, P., Tang, C. \& Wiensenfeld, K., 1988. Self-organized criticality. Physical Review A, 38(1), pp.364-375.

Bédard, C., Kröger, H. \& Destexhe, a., 2006. Does the $1 / f$ frequency scaling of brain signals reflect self-organized critical states? Physical Review Letters, 97(11), pp.1-4.

El Boustani, S. et al., 2009. Network-state modulation of power-law frequency-scaling in visual cortical neurons. PLoS Computational Biology, 5(9).

Börgers, C. \& Kopell, N.J., 2008. Gamma oscillations and stimulus selection. Neural computation, 20(2005), pp.383-414.

Chaudhuri, R., He, B.J. \& Wang, X., 2016. Random recurrent networks near criticality capture the broadband power distribution of human ECoG dynamics. , 62349, pp.1-47.

Cox, R. et al., 2016. Variability and stability of large scale cortical oscillatory patterns. bioRxiv.

Crespo-Facorro, B. et al., 2001. Neural Mechanisms of Anhedonia in Schizophrenia: A PET Study of Response to Unpleasant and Pleasant Odors. JAMA, 286(4), pp.427-435.

Dehghani, N. et al., 2016. Dynamic Balance of Excitation and Inhibition in Human and Monkey Neocortex. Scientific Reports, 6(23176), pp.1-12.

Destexhe, A. \& Rudolph, M., 2004. Extracting information from the power spectrum of synaptic noise. Journal of computational neuroscience, 17(3), pp.327-345.

First, Michael B., Spitzer, Robert L, Gibbon Miriam, and Williams, Janet B.W.: Structured Clinical Interview for DSM-IV-TR Axis I Disorders, Research Version, Patient Edition. 
(SCID-I/P) New York: Biometrics Research, New York State Psychiatric Institute, November 2002.

Fischler, Martin a., and Robert C. Bolles. 1981. "Random Sample Consensus: A Paradigm for Model Fitting with Applications to Image Analysis and Automated Cartography." Communications of the ACM 24 (6): 381-95. doi:10.1145/358669.358692.

Freeman, W.J. \& Zhai, J., 2009. Simulated power spectral density (PSD) of background electrocorticogram (ECoG). Cognitive Neurodynamics, 3, pp.97-103.

Gao, R., Peterson, E.J. \& Voytek, B., 2016. Inferring Synaptic Excitation/Inhibition Balance from Field Potentials. bioRxiv, pp.1-31.

Guntupalli, J.S. et al., 2016. A Model of Representational Spaces in Human Cortex. Cerebral Cortex, 26(6), pp.2919-2934.

Hermes, D., Miller, K. J., Wandell, B. A. \& Winawer, J. Stimulus Dependence of Gamma Oscillations in Human Visual Cortex. Cereb Cortex (2014).

Jung, Tzyy-Ping P, Scott Makeig, Colin Humphries, Te-Won W Lee, Martin J McKeown, Vicente Iragui, and Terrence J Sejnowski. 2000. "Removing Electroencephalographic Artifacts by Blind Source Separation." Psychophysiology $37 \quad$ (2): 163-78. doi:10.1111/1469-8986.3720163.

Kanwisher, N., 2010. Functional specificity in the human brain: a window into the functional architecture of the mind. Proceedings of the National Academy of Sciences of the United States of America, 107, pp.11163-11170.

Kendal, W.S., 2013. Fluctuation Scaling and 1/f Noise. Journal of Basic and Applied Physics, 2(May), pp.40-49.

Lee, K. et al., 2010. Gamma ( $40 \mathrm{~Hz}$ ) phase synchronicity and symptom dimensions in schizophrenia. Cognitive Neuropsychiatry, (October 2013), pp.37-41. 
Makeig, Scott, Anthony J. Bell., Tzyy-Ping Jung, and Terrence J. Sejnowski. 1996. “Independent Component Analysis of Electroencephalographic Data." Advances in Neural Information Processing Systems 8: 145-51. doi:10.1109/ICOSP.2002.1180091.

Mandelbrot, B.B. \& Van Ness, J.W., 1968. Fractional Brownian Motions, Fractional Noises and Applications. SIAM Review, 10(4), pp.422-437.

Merker, B., 2013. Cortical gamma oscillations: the functional key is activation, not cognition. Neuroscience and biobehavioral reviews, 37(3), pp.401-17.

Miller, K.J., 2010. Broadband spectral change: evidence for a macroscale correlate of population firing rate? The Journal of neuroscience : the official journal of the Society for Neuroscience, 30(19), pp.6477-9.

Milstein, J. et al., 2009. Neuronal shot noise and Brownian 1/f2 behavior in the local field potential. PloS one, 4(2), p.e4338.

Perlstein, W.M. et al., 2001. Relation of Prefrontal Cortex Dysfunction to Working Memory and Symptoms in Schizophrenia. Am J Psychiatry, 158(7), pp.1105-1113.

Phillips, W. a \& Silverstein, S.M., 2003. Convergence of biological and psychological perspectives on cognitive coordination in schizophrenia. The Behavioral and brain sciences, 26(1), pp.65-82-137.

Podvalny, E. et al., 2015. A unifying principle underlying the extracellular field potential spectral responses in the human cortex. Journal of Neurophysiology, p.jn.00943.2014. Available at: http://jn.physiology.org/lookup/doi/10.1152/jn.00943.2014.

Welch, Peter D. 1967. "The Use of Fast Fourier Transform for the Estimation of Power Spectra: A Method Based on Time Averaging Over Short, Modified Periodograms." IEEE Transactions on Audio and Electroacoustics $15 \quad$ (2): 70-73. doi:10.1109/TAU.1967.1161901. 
R Core Team, 2016. R: A Language and Environment for Statistical Computing. Available at: https://www.r-project.org/.

Salthouse, T.A. \& Lichty, W., 1985. of the Neural Noise Hypothesis Cognitive Change ' ;“ J : T : Jrj :.:":. J '. , 40(4), pp.443-450.

Ray, S., Ni, A.M. \& Maunsell, J.H.R., 2013. Strength of Gamma Rhythm Depends on Normalization. PLoS Biology, 11(2).

Ray, S. \& Maunsell, J.H.R., 2010. Differences in gamma frequencies across visual cortex restrict their possible use in computation. Neuron, 67(5), pp.885-96.

Sun, Y. et al., 2011. Gamma oscillations in schizophrenia: Mechanisms and clinical significance. Brain Research, 1413, pp.98-114.

Uhlhaas, P.J. \& Singer, W., 2010. Abnormal neural oscillations and synchrony in schizophrenia. Nature reviews. Neuroscience, 11(2), pp.100-113.

Uhlhaas, P.J. \& Singer, W., 2012. Neuronal Dynamics and Neuropsychiatric Disorders: Toward a Translational Paradigm for Dysfunctional Large-Scale Networks. Neuron, 75(6), pp.963-980.

Voytek, B. \& Knight, R.T., Dynamic network communication as a unifying neural basis for cognition, development, aging, and disease. 2016, pp.1-20.

Voytek, B. et al, 2015. Age-related Changes in 1/F. J Neuro, Pre-pub, pp.1-25.

Wang, X.-J., 2010. Neurophysiological and computational principles of cortical rhythms in cognition. Physiological reviews, 90(3), pp.1195-268.

Waschke, L., Wöstmann, M. \& Obleser, J., 2017. Neural noise in the age-varying human brain predicts perceptual decisions. bioRxiv, pp.1-42. 
Winterer, G. \& Weinberger, D.R., 2003. Cortical signal-to-noise ratio: Insight into the pathophysiology and genetics of schizophrenia. Clinical Neuroscience Research, 3(1-2), pp.55-66.

Womelsdorf, T. et al., 2007. Modulation of neuronal interactions through neuronal synchronization. Science (New York, N.Y.), 316(June), pp.1609-1612.

Womelsdorf, T. et al., 2014. Dynamic circuit motifs underlying rhythmic gain control, gating and integration. Nature Neuroscience, 17(8), pp.1031-1039.

Wöstmann, L., Malte, W. \& Obleser, J., 2017. Neural noise in the age-varying human brain predicts perceptual decisions. bioRxiv, pp.1-34. 